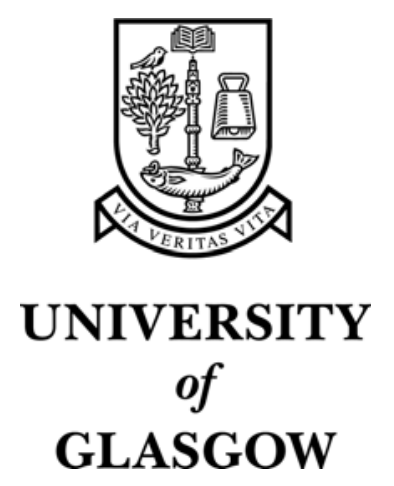

Rasmussen, K.F. and Davies, J.H. and Miller, T.J.E. and McGilp, M.I. and Olaru, M. (2000) Analytical and numerical computation of air-gap magnetic fields in brushless motors with surface permanent magnets. IEEE Transactions on Industry Applications 36(6):pp. 1547-1554.

http://eprints.gla.ac.uk/archive/00002837/ 


\title{
Analytical and Numerical Computation of Air-Gap Magnetic Fields in Brushless Motors with Surface Permanent Magnets
}

\author{
Keld Folsach Rasmussen, Member, IEEE, John H. Davies, T. J. E. Miller, Fellow, IEEE, Malcolm Iain McGilp, \\ and Mircea Olaru
}

\begin{abstract}
This paper extends the theory of the air-gap magnetic field in permanent-magnet (PM) brushless motors. Scalar and vector potential solutions to the field equations are brought together to unify many of the important practical methods already in use. The theory admits a more general representation of the magnetization vector than has been previously assumed, including both the radial and tangential components, and variation with radius. The work is applied in the design of PM motors where there is a requirement to minimize noise and torque ripple, and maximize efficiency, and a continuing need for improvements in the accuracy and rigor of design calculations. The air-gap flux-density distribution is at the heart of the design process, and it is desirable to study different magnetization patterns, including imperfections in the magnetization, for a wide range of magnet shapes. This paper shows the application of the analytical solutions in comparison with a new finite-element procedure, with test results on a prototype motor, and with simpler, older methods of calculation based on magnetic equivalent circuits. The comparison brings out many interesting points in relation to the accuracy and the speed and practicality of the various methods.
\end{abstract}

Index Terms-Magnetic analysis, permanent-magnet brushless machines.

\section{INTRODUCTION}

$\mathbf{P}$ ERMANENT-MAGNET motors of both brushed and brushless types are widely used in automotive, industrial, and other applications where their simplicity, high efficiency, linearity, and smooth, quiet operation are advantageous. Accordingly, there is much new design activity, in which there is pressure to improve the design and to produce prototypes as quickly as possible on a "right first time" basis. Significant developments are taking place in relation to noise and torque ripple, which are important in applications such as power steering, but may be equally important in liquid pumping

Paper IPCSD 99-109, presented at the 1999 Industry Applications Society Annual Meeting, Phoenix, AZ, October 3-7, and approved for publication in the IEEE TRANSACTIONS ON INDUSTRY APPLICATIONS by the Electric Machines Committee of the IEEE Industry Applications Society. Manuscript submitted for review June 1, 1999 and released for publication July 4, 2000. This work was supported by the Danish Energy Research Programme and the SPEED Consortium.

K. F. Rasmussen is with Grundfos A/S, 8850 Bjerringbro, Denmark (e-mail: kfolsach@grundfos.com).

J. H. Davies, T. J. E. Miller, and M. I. McGilp are with the SPEED Laboratory, University of Glasgow, Glasgow, G12 8LT, U.K. (e-mail: T.Miller@elec.gla.ac.uk).

M. Olaru is with ICNDMF-CEFIN, 70314 Bucharest, Romania (e-mail molaru@ hades.ro).

Publisher Item Identifier S 0093-9994(00)10428-1. applications where the resonances of a piping system can be excited [1].

The techniques for achieving smooth, quiet operation include traditional methods such as the use of skewed magnets or armature slots; and the correct choice of slots/pole, winding distribution, and magnet arc. The magnet shape has always been recognized as an important factor, but the variation of the magnetization within the magnet is equally important and is recognized in the recent interest in Halbach arrays and other configurations, [2], [3]. The preferred method for analyzing these effects has been the finite-element (FE) method [4], but this lacks the theoretical framework of classical motor theory and leads to an essentially experimental approach to the design optimization.

Zhu et al. [5] presented an analytical solution of the magnetic field in both interior-and exterior-rotor permanent-magnet (PM) motors, including the superimposed effect of armature current and the influence of slotting. This work was validated by physical test and by comparison with FE analysis. More recently, Kim and Lieu [6] extended Zhu's work to the case of an eccentric rotor, also including the effect of slotting. These papers provide a direct analytical solution to the air-gap field, which previously had been approximated using semi-empirical methods [7], [8]. However, they excluded the tangential component of magnetization and restricted their attention to magnets that were magnetized wholly in the radial direction.

In many modern designs, these assumptions are not justified. For example, if the magnet is magnetized in situ, the tangential component of magnetization $M_{\theta}$ is unavoidable [1] and, indeed, it may be incorporated by design, for it is known to be capable of improving the harmonic content of the electromotive force (EMF) waveform and even of increasing the peak air-gap flux density, compared with that of the radially magnetized magnet [1], [9]. These properties are demonstrated in [1], which extends the analysis along Zhu's original lines, to incorporate magnets having a wider range of magnetization patterns including the tangential component.

Until Zhu's paper, it appears that only Boules [9], [10] had a solution of comparable analytical power, that would deal with more general patterns of magnetization including $M_{\theta}$, for example, the important practical case of a parallel-magnetized magnet. Boules represented the magnets by equivalent distributions of current sheets across their surfaces. Related analyses of comparable complexity had been published earlier by Hughes and Miller [11] in relation to slotless superconducting synchronous machines, but their solutions were in terms of 
Fourier series of sinusoidally distributed windings rather than of distributions of current filaments or sheets, and were perhaps not obviously adaptable to the PM machine, in spite of the affinity between these types of machine.

Recent advances in the design of PM brushless motors, and competitive pressure to reduce noise and torque ripple, have generated a need for even more advanced solutions and, in particular, the variation of magnetization throughout the material has become an important consideration. This variation was neglected in most earlier work, but see [17]-[21]. Even in the lumped-parameter magnetic equivalent-circuit approach, the problem arises in relation to the dimensions that should be used for the internal permeance of an arcuate magnet-whether to use the mean radius or some other radius closer to one surface.

This paper presents a new analysis of the PM field in which both the radial and tangential components of magnetization are present, and radial variation is accommodated by means of a Green function (equivalent to a variation of parameters). Moreover, the solution is developed in alternative ways, one in terms of a scalar potential, continuing the work of Rasmussen [1], and the other in terms of a vector potential. By this means, the various prior theories are unified, and the vector potential solution is shown to lead to Hague's solution [12] for the distribution of equivalent current filaments, which was used by Boules [10]. Furthermore, Boules' method itself is extended to a wider range of magnet geometries, throwing considerable light on the choice of the most appropriate method in particular circumstances. Taken together with [1] for the case of magnets having a relative recoil permeability different from 1 , the paper can perhaps claim the most general solution to date for this problem in cylindrical coordinates, and it unifies theoretical approaches which up to now have appeared to be unrelated.

Since the vector potential solution is developed in terms of an equivalent distribution of ampere conductors, it can also be used to compute the armature-reaction field of the stator currents. In fact, this procedure has been used for a long time and it is also developed for complete arrays of conductors, leading to the expressions for the classical distribution factor and pitch factor; see, for example, [12].

A further result is to test some of the earlier semi-empirical methods [7], [8], which are shown to have surprisingly good results in spite of their simplicity.

The main limitation of the method is that it neglects saturation and slotting, but it is still worthwhile to develop improved analytical solutions. Most of the motors for which the analysis was developed are typical brushless PM motors with semi-closed slots, in which the effect of the slot openings is relatively small for most aspects of performance except cogging torque. In many cases, the analytical method is sufficiently accurate and, where the effects of saturation and slotting are significant, the FE method can be used. Skewing, however, is included in the analytical solution.

The methods have been tested by physical measurements on prototype motors (see Fig. 1). In addition, extensive testing against FE methods has been undertaken using a specially developed FE procedure coupled to a motor design program. Provided that the motor has sufficient symmetry, the FE method

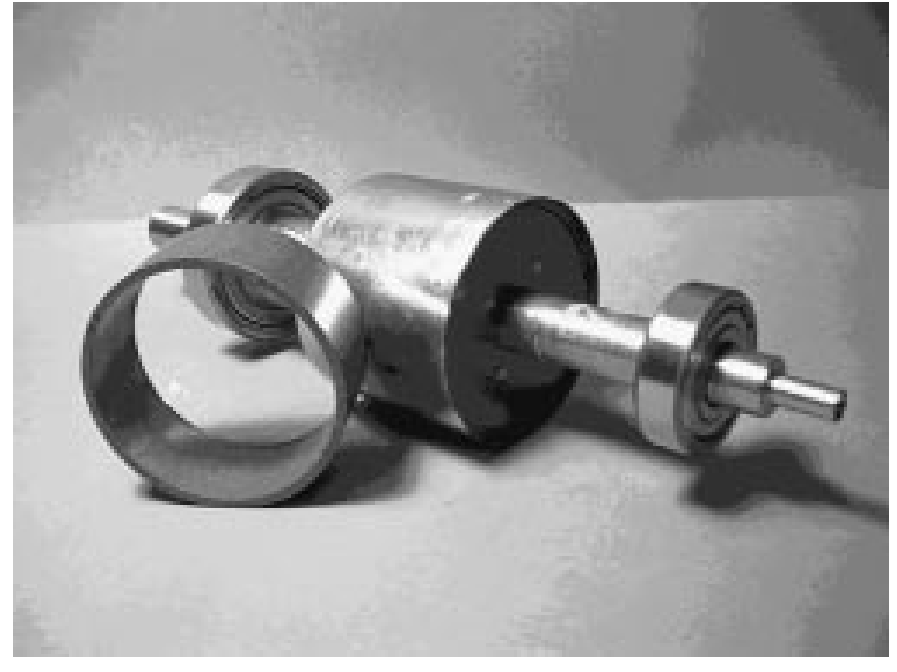

Fig. 1. Typical PM brushless motor rotor, with full-ring magnet.

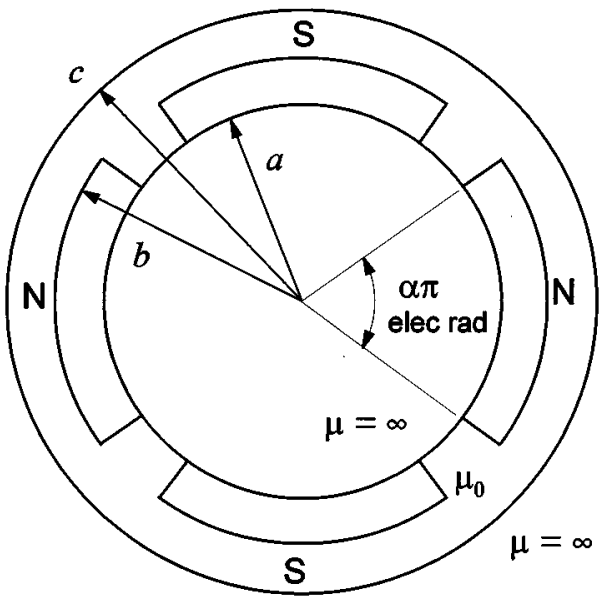

Fig. 2. Layout and dimensions of the motor.

can be quicker than the new analytical methods when the field is calculated over several radii to give a complete view, but when calculating at one radius (usually the stator bore), the analytical methods are much faster. However, improvements in speed would still be valuable. The analytical methods rely on Fourier series expansions of the field components, which are built into the original separation of the variables applied to the solution of the partial differential field equation. The series converge slowly, and their coefficients are complicated with large powers of ratio of radii. It remains as a research challenge to find ways to accelerate this convergence possibly by means of an alternative representation of the azimuthal variation of the field.

\section{THEORY}

Fig. 2 shows the configuration of a typical interior-rotor brushless PM motor. The theory applies equally to the exterior-rotor configuration. The main assumptions are as follows.

1) Both the rotor steel $(r<a)$ and the stator steel $(r>c)$ are assumed infinitely permeable. 
2) Slotting is neglected.

3) The magnet operates on a recoil line whose relative permeability is assumed to be unity.

The third assumption is equivalent to writing $\mathbf{B}=\mu_{0} \mathbf{H}+\mathbf{M}$ and taking $\mathbf{M}$ to be entirely the specified magnetization in the magnets, with no further contribution from $\mathbf{H}$. This simplification was not made in [1], but Boules has shown that the error is typically only $1 \%$ or $2 \%$ with modern magnets [9]. The principal extension of the theory is to allow the magnetization to be an arbitrary function within the region $a<r<c$. The development is given for the vector potential because this leads directly to the equivalent-coil method discussed in Section III. The complementary scalar-potential solution is given in [1].

The magnetization $\mathbf{M}$ is replaced by a volume current density $\mathbf{J}=\left(1 / \mu_{0}\right) \operatorname{curl} \mathbf{M}\left[\mathrm{A} / \mathrm{m}^{2}\right]$ in the bulk and a linear current density $\mathbf{K}=\left(1 / \mu_{0}\right) \mathbf{M} \times \mathbf{n}[\mathrm{A} / \mathrm{m}]$ on the surfaces of the magnets. We assume as usual that the motor extends infinitely along $z$, so all currents flow in the axial direction and the vector potential also reduces to $A_{z}$. Thus, we have to solve only the scalar Poisson equation $\nabla^{2} A_{z}=-\mu_{0} J_{z}$. As in [1], we expand all quantities in Fourier series.

The magnet lies within an annular region and its magnetization vector is

$$
\mathbf{M}=M_{r} \hat{r}+M_{\theta} \hat{\theta}
$$

where $M_{r}$ and $M_{\theta}$ are defined by

$$
\begin{aligned}
& M_{r}(r, \theta)=\sum_{n} M_{r, n}(r) \cos n p \theta \\
& M_{\theta}(r, \theta)=\sum_{n} M_{\theta, n}(r) \sin n p \theta
\end{aligned}
$$

where $p$ is the number of pole pairs and $n$ is the harmonic number. Both radial and tangential components of the magnetization are included. The Fourier series for any source current distribution is

$$
J_{z}(r, \theta)=\sum_{n} J_{n}(r) \sin n p \theta .
$$

The magnetic flux density $B_{r}, B_{\theta}$ and vector potential $A_{z}$ are expanded in the same way as $M_{r}, M_{\theta}$ and $J_{z}$, respectively.

The Fourier components of the current density are given in terms of the magnetization by

$$
\mu_{0} J_{n}=\frac{1}{r} \frac{d}{d r}\left(r M_{\theta, n}\right)+\frac{n p}{r} M_{r, n} .
$$

Finally, having found the vector potential, the flux density follows from

$$
B_{r, n}=\frac{n p A_{n}}{r} \quad B_{\theta, n}=-\frac{d A_{n}}{d r} .
$$

The general solution for each Fourier component of the vector potential can be written as an integral over the current density using a Green function,

$$
A_{n}(r)=\int_{a}^{c} G_{n}\left(r, r^{\prime}\right) \mu_{0} J_{n}\left(r^{\prime}\right) d r^{\prime}
$$

The Green function is the solution to Poisson's equation for a single sheet of current at radius $r^{\prime}$ and obeys the full boundary conditions. It is convenient to assume that there is an infinitesimal air gap between the magnets and the infinitely permeable rotor and stator, in which case the boundary conditions are that $B_{\theta}=0$ at $r=a$ and $r=c$; this requires careful treatment of the surfaces of the magnets, which will be discussed below. The Green function then takes the form

$$
G_{n}\left(r, r^{\prime}\right)=\frac{r^{\prime}}{2 n p} \frac{u_{n p<}\left(r_{<}\right) u_{n p>}\left(r_{>}\right)}{(c / a)^{n p}-(a / c)^{n p}} .
$$

The notation $r_{<}$and $r_{>}$means the lesser and greater of $r$ and $r^{\prime}$. The Green function is not analytic at $r=r^{\prime}$, where there is a discontinuity in the slope induced by the sheet of current. Finally, the functions $u_{n p}<$ and $u_{n p}>$ are solutions to Laplace's equation that obey the boundary conditions at the lower and upper boundaries, respectively, and are given by

$$
\begin{aligned}
& u_{n p<}(r)=(r / a)^{n p}+(a / r)^{n p} \\
& u_{n p>}(r)=(r / c)^{n p}+(c / r)^{n p} .
\end{aligned}
$$

This solution holds for $n p \geq 1$ and could also have been obtained by the variation of parameters. A similar expression can be derived for the magnetic scalar potential, with the magnetic charge density $\rho=-\operatorname{div} \mathbf{M}$ as source rather than the current $\mathbf{J}$; the functions in (8) are replaced by expressions that obey the boundary conditions for $\mathbf{H}$ rather than $\mathbf{B}$ [1].

The Green function contributes only powers of $r^{\prime}$ to the integral in (6), so it should be possible to evaluate this analytically for many forms of radial variation in the magnetization.

For example, a radially magnetized magnet with remanent flux density $B_{r}$ and arc $\alpha \pi$ electrical radians (as in Fig. 2) has

$$
M_{n, r}=\frac{4 B_{r}}{n \pi} \sin (n \pi \alpha / 2) \quad M_{n, \theta}=0
$$

for $n=1,3,5, \cdots, \infty$. Care must be taken when dealing with surfaces of the magnets, however, including surfaces adjacent to the rotor and stator. These surfaces generate equivalent currents of the form $\mu_{0} \mathbf{K}=\mathbf{M} \times \mathbf{n}$, which provide singular contributions to the (volume) current density in (6). For instance, a Fourier component $K_{n}$ due to a surface at $r=b$ must be included in the integral as $J_{n}(r)=K_{n} \delta(r-b)$. The $\delta$ function causes the integral over the Green function to collapse, giving simple contributions to the vector potential. This will be seen in the example to be considered next.

Piecewise Constant Magnetization as a Function of Radius: As a first example, we consider the problem solved by Rasmussen [1]. This has a full magnetic ring on the rotor surface, $a<r<b$, with an air gap $b<r<c$. The magnetization is independent of radius in [1] and the Fourier components are

$$
M_{r, n}=M_{n} \quad M_{\theta, n}=N_{n}
$$

for $a<r<b$, and zero for $b<r<c$. The integration over the bulk in (6) is straightforward, but we must not forget the inner and outer interfaces of the magnet, where surface currents are generated by the tangential component of magnetization. The inner edge at $r=a$ contributes $\mu_{0} K_{n}=N_{n}$ while the outer edge at $r=b$ contributes $\mu_{0} K_{n}=-N_{n}$. The two give a 
current density of $\mu_{0} J_{n}^{(\text {interfaces })}=N_{n}[\delta(r-a)-\delta(r-b)]$. The $\delta$ functions make the integrals over the Green function in (6) trivial, and produce a contribution to the vector potential of

$$
A_{n}^{\text {(interfaces) }}(r)=N_{n}\left[G_{n}(r, a)-G_{n}(r, b)\right] .
$$

After performing the integration over the bulk, the total vector potential in the air gap $b<r<c$ is found to be

$$
A_{n}(r)=\frac{b / 2}{1-(n p)^{2}}\left[\frac{(r / c)^{n p}+(c / r)^{n p}}{(a / c)^{n p}-(c / a)^{n p}}\right]\left(M_{n} U+N_{n} V\right)
$$

where

$$
\begin{aligned}
& U=2 a / b+(n p-1)(b / a)^{n p}-(n p+1)(a / b)^{n p} \\
& V=2 n p a / b-(n p-1)(b / a)^{n p}-(n p+1)(a / b)^{n p} .
\end{aligned}
$$

These expressions hold for $n p>1$; logarithms appear for $n p=$ 1 . The dependence on $r$ comes entirely from $u_{n p}>(r)$, which confirms that the function satisfies the boundary condition on the surface of the stator at $r=c$. The resulting flux density agrees with [1] after setting $\mu_{r}=1$ there.

Single Conductor: The second example leads to the equivalent-coil approach. We start by concentrating the equivalent current density from the magnetization into a few discrete current filaments. The magnetic field can then be deduced from the results of Hague [12], who derives the magnetic field due to distributions of conductors in an annular air gap. To make contact with his result for a single conductor, consider a wire in the air gap at $r=b$ and $\theta=0$ that carries current $I$ along the $+z$ axis. This is equivalent to a current density

$J_{z}(r, \theta)=\frac{I \delta(r-b) \delta(\theta)}{b}=\frac{I \delta(r-b)}{\pi b}\left[\frac{1}{2}+\sum_{n=1}^{\infty} \cos n \theta\right]$

Note that this is a sum of cosines, rather than sines as in (1). The constant component in the Fourier series $(n=0)$ has no practical impact because a balanced set of magnets generates no net current density and the final result must, therefore, be summed over a set of current filaments whose total is zero.

The calculation of the vector potential is straightforward because of the $\delta$ function in the current density, and we find

$$
\begin{aligned}
A_{z}(r) & =\sum_{n=1}^{\infty} \cos n \theta \int_{a}^{c} G_{n}\left(r, r^{\prime}\right) \mu_{0} J_{n}\left(r^{\prime}\right) d r^{\prime} \\
& =\frac{\mu_{0} I}{\pi b} \sum_{n=1}^{\infty} \cos n \theta \int_{a}^{c} G_{n}\left(r, r^{\prime}\right) \delta\left(r^{\prime}-b\right) d r^{\prime} \\
& =\frac{\mu_{0} I}{2 \pi} \sum_{n=1}^{\infty} \frac{(1 / n) \cos n \theta}{(c / a)^{n}-(a / c)^{n}} \mid \begin{array}{l}
u_{n>}(b) u_{n<}(r), a<r<b \\
u_{n<}(b) u_{n>}(r), b<r<c
\end{array}
\end{aligned}
$$

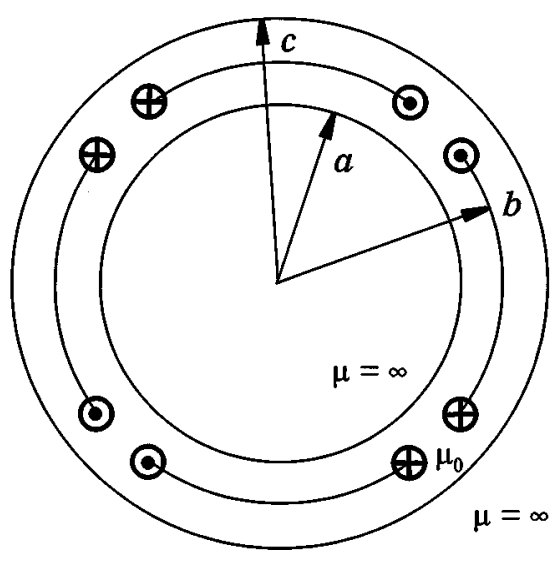

Fig. 3. Set of $2 p$ filamentary coils.

where $u_{n}<$ and $u_{n}>$ are given by (8). The flux density is deduced using $B_{r}=(1 / r) \partial A / \partial \theta$ and $B_{\theta}=-\partial A / \partial r$. After some manipulation, the results for $r>b$ reduce to

$$
\begin{aligned}
& B_{r}=-\frac{\mu_{0} I}{2 \pi r} \sum_{n=1}^{\infty} \frac{c^{n}}{b^{n}} \frac{b^{2 n}+a^{2 n}}{c^{2 n}-a^{2 n}}\left[\frac{r^{n}}{c^{n}}+\frac{c^{n}}{r^{n}}\right] \sin n \theta \\
& B_{\theta}=-\frac{\mu_{0} I}{2 \pi r} \sum_{n=1}^{\infty} \frac{c^{n}}{b^{n}} \frac{b^{2 n}+a^{2 n}}{c^{2 n}-a^{2 n}}\left[\frac{r^{n}}{c^{n}}-\frac{c^{n}}{r^{n}}\right] \cos n \theta .
\end{aligned}
$$

These are consistent with Hague [12] apart from the omission of the term with $n=0$.

\section{EQuivalENT-CoIL MeTHOD}

The equivalent-coil method (Fig. 3) uses Hague's analysis [12]. While the magnets are represented by equivalent distributions of surface current $\mathbf{K}=\left(1 / \mu_{0}\right) \mathbf{M} \times \mathbf{n}$ and volume current $\mathbf{J}=\left(1 / \mu_{0}\right) \operatorname{curl} \mathbf{M}$, the total field requires an integration of the effect of both $\mathbf{K}$ and $\mathbf{J}$ over the entire cross section of the motor. Boules did the integration analytically for radially magnetized and parallel-magnetized magnets with $J=0$ [9], but the integration is intractable in all but the simpler cases. However, such is the power of modern desktop computers, that it is now possible to replace both $\mathbf{K}$ and $\mathbf{J}$ by a discrete set of filamentary coils and simply accumulate the total effect by a summation process. Fig. 4 shows, diagrammatically, the nature of the distribution of filamentary coils for a common magnet configuration used in dc motors, for which the integration of the effect of $\mathbf{K}$ is feasible but tedious.

The number of filaments can be limited to a reasonable number for computation, because higher order space harmonics decay quickly away from the magnet surfaces.

In Fig. 3, a basic four-pole distribution of single-turn coils has radius $c$ and span $2 \xi$, centered on the $x$ axis, and the radial component of flux density at the point $(r, \theta)$ is given by

$$
\begin{aligned}
B_{r}=\frac{2 p \mu_{0} I}{\pi r} \sum_{n}^{\infty} \frac{c^{n}}{b^{n}} \frac{b^{2 n}+a^{2 n}}{c^{2 n}-a^{2 n}}\left[\frac{r^{n}}{c^{n}}+\frac{c^{n}}{r^{n}}\right] \\
k_{\sigma n} \sin n \xi \cos n \theta
\end{aligned}
$$




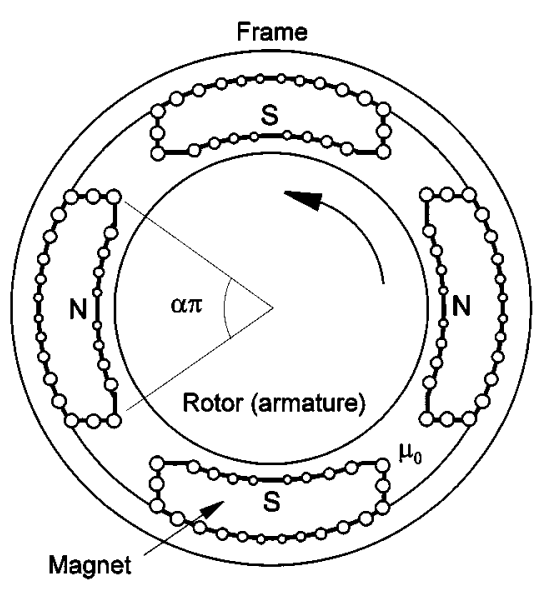

Fig. 4. Distribution of current filaments.

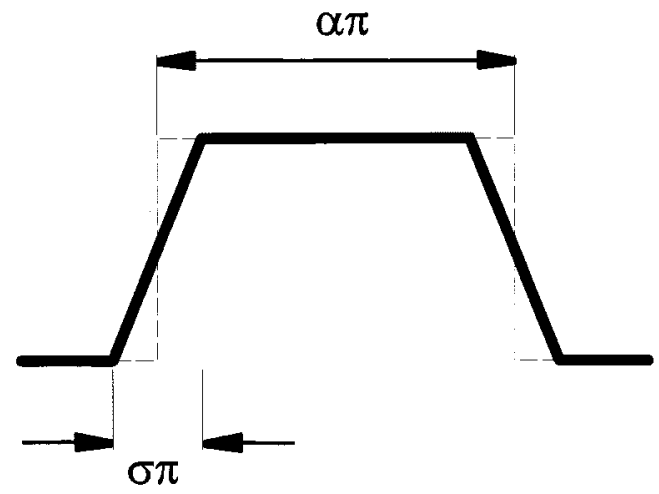

Fig. 5. Tapered magnetization. The effect is similar to that of a skew of $\sigma \pi$.

where $I$ is the coil current, $p$ is the number of pole pairs, and the sum is taken with $n=(2 j-1), j=1,2,3 \cdots ;$ i.e., over all odd electrical harmonics [12].

The factor $k_{\sigma n}$ is the $n$th harmonic skew factor equal to $\sin (n \sigma \pi / 2) /(n \sigma \pi / 2)$, where $\sigma \pi$ is the skew in electrical radians (Fig. 5). When the contributions of all the coils are summed, the same factor arises if the magnetization tapers from a peak value to zero over an angle $\sigma \pi$ electrical radians, proving that tapered magnetization and skew are equivalent in terms of their effect on the air-gap field. The same factor arises in the generated EMF of a lap winding having a phase spread of $\sigma \pi$ electrical radians.

If the poles are unevenly magnetized, the field can be calculated on a pole-by pole basis, and even-order harmonics arise. The method can also be used to calculate the air-gap component of the phase inductance for any distribution of coils, as has been recently described for dc commutator motors, [15].

In both analytical methods, the field expressions require efficient programming to minimize computation time. The radii are normalized to avoid generating very large numbers when $n$ is large. Calculations are performed in double precision (about 20 significant figures). The expressions are inherently ill conditioned when $n$ is large, but, fortunately there is no need to calculate more harmonics than are likely to be effective in the machine. Even a small degree of skew or magnet taper filters out the high-order harmonics, and often there is no need to calcu-

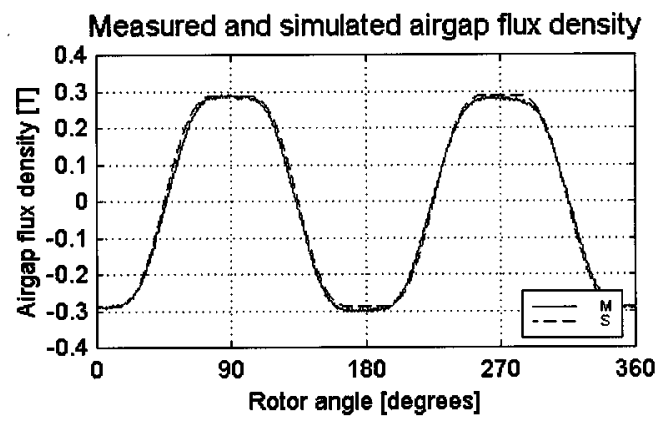

Fig. 6. Air-gap flux-density distribution around the airgap, comparing measurement against the scalar potential calculation method.

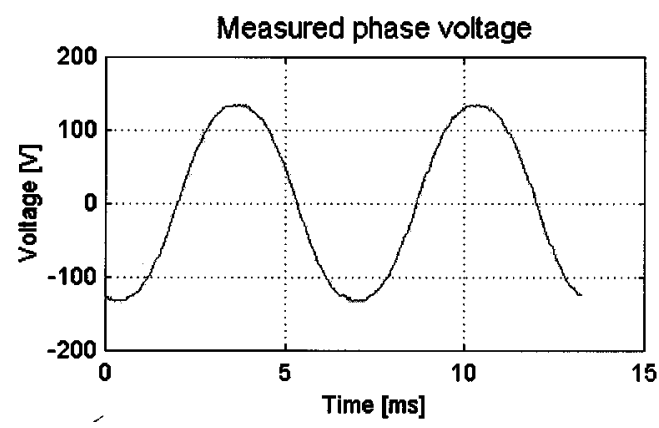

Fig. 7. Measured back-EMF waveform; the calculated wave is indistinguishable from the measured one at this scale.

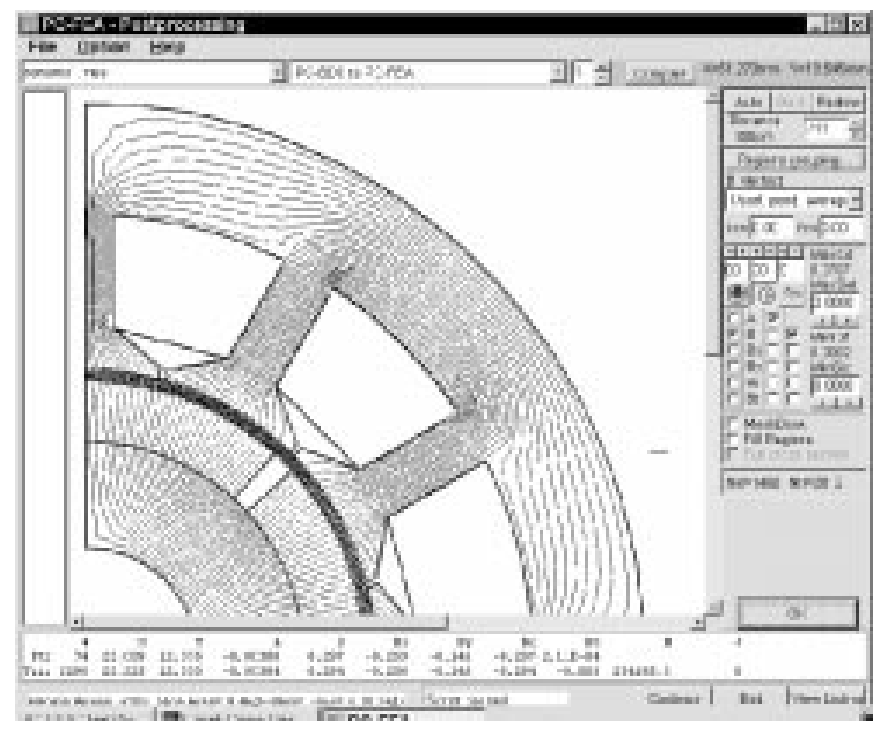

Fig. 8. Finite-element flux plot for the test motor in Fig. 8.

late more than about 20 harmonics (i.e., up to the 39th physical harmonic in a four-pole motor: $n p=2 \times 20-1)$.

\section{TYPICAL RESULTS}

The calculations were compared against physical measurements on a small prototype brushless PM motor. Fig. 6 shows measured and calculated air-gap flux density, and Fig. 7 shows the back-EMF waveform when the motor is rotated on open circuit. The agreement is very close. Additional test data on the harmonic content of these waveforms are given in [1]. 


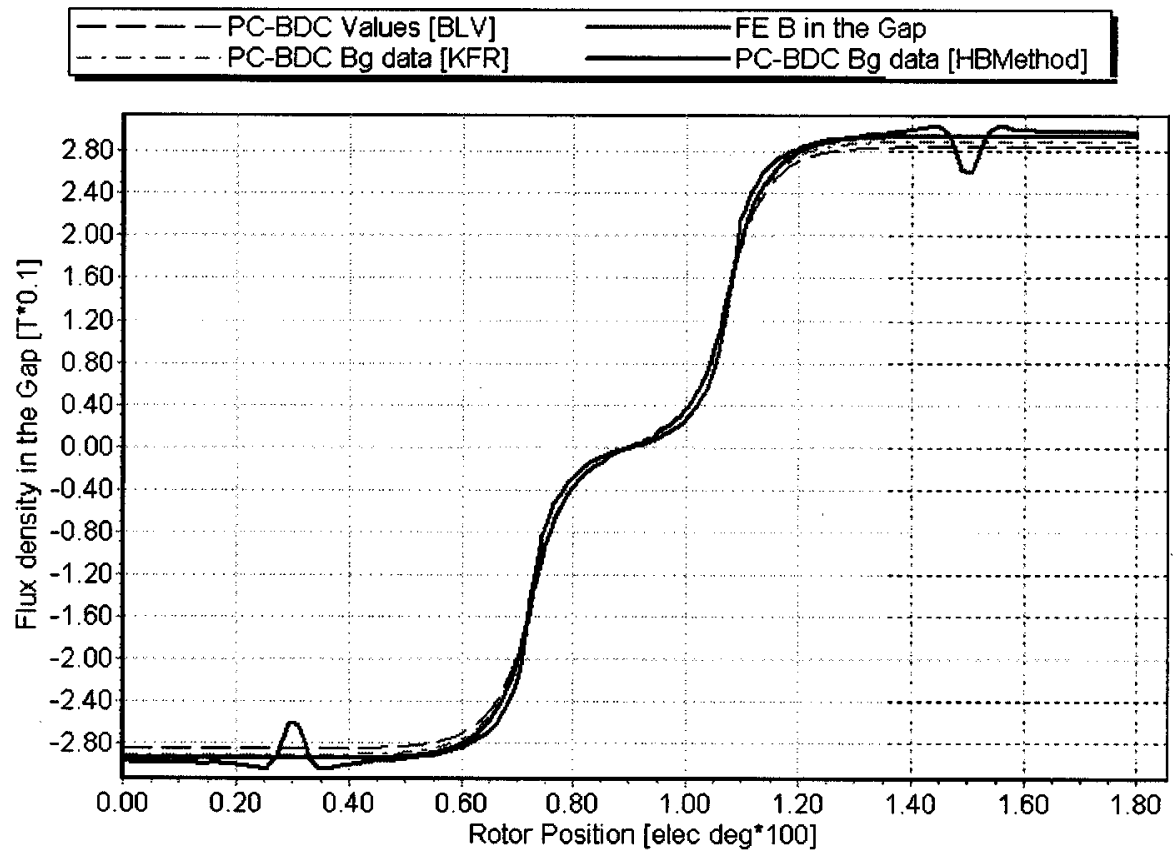

Fig. 9. Air-gap flux-density calculated by three analytical methods, and by FE analysis.

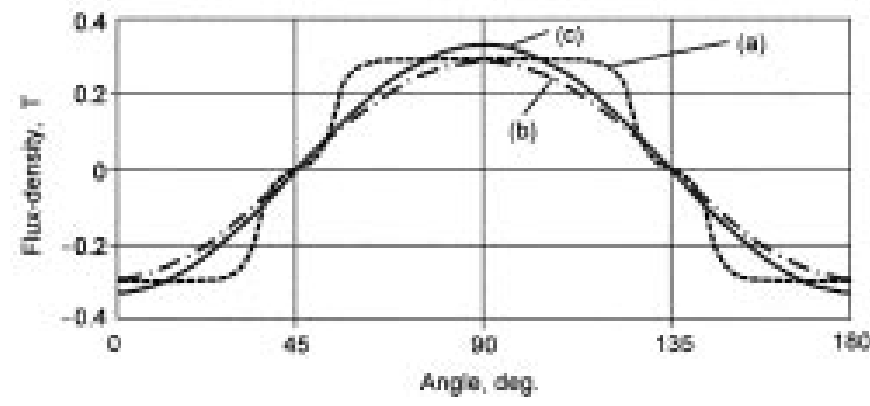

Fig. 10. Air-gap flux-density distribution for (a) radial magnetization, (b) radial sine magnetization (no tangential component), and (c) full sine magnetization.

The new calculation methods were also compared with a number of older published methods and against FE computations (Figs. 8 and 9) [16]. Fig. 9 shows the air-gap flux-density waveform computed by three analytical methods and the FE method. The three analytical methods are as follows:

1) Miller and Rabinovici's method as described in [7];

2) the scalar potential method described in [1];

3) the equivalent-coil method described in Section III.

Only the FE method models slotting and saturation, but in open-circuit calculations this is often insignificant, particularly at the initial design stage. The three analytical methods agree closely, even though the magnet has a recoil permeability of 1.1, which works against the equivalent-coil method.

Fig. 10 shows the comparison between different magnetization configurations. It is often held that sine-wave magnetization produces a lower air-gap flux density than radial magnetization, but in the example in Fig. 10, the full sine-wave magnetization has the highest value due to the flux focusing associated with the tangential component.
It can be observed that, since the method is linear, the effect of nonsymmetrical placement of magnets can be calculated by superposition of the fields of individual magnets, or by adding a "perturbation" calculated for a fictitious magnet which represents the difference in magnetization distribution between the actual magnet and the ideal arrangement of magnets. The perturbation can be represented by odd or even harmonics, or both, according to the actual disposition of the magnets.

Practical Comparison of Methods: Although the methods agree for a motor of conventional proportions, they are not all equally fast. The software environment for the calculations (including the FE method) was the SPEED Laboratory motor design software [14] and [16]. As expected, the magnetic-circuit model is the fastest, but it is also the least rigorous and, if large changes were made to the proportions, it could become inaccurate, while the new analytical methods would need no adjustment. The FE method might also require adjustments to the mesh before a good solution could be obtained. For the test calculations, it could calculate the entire field in about the same time that the new analytical method would require for ten radii, but motor design calculations generally require the field at only one or two radii, often with only a limited number of harmonics. With a fractional slot motor, the FE method would be even slower.

\section{CONCLUSION}

This paper has presented an extension to the theory of the air-gap field in PM motors (both brushless and brushed), in which both the variation of magnetization with radius, and the tangential component of magnetization, are taken into account. Solutions were developed in terms of both scalar and vector potentials, and shown to be equivalent. The vector potential solution is shown to lead directly to the equivalent-coil method used 
by Boules, [9]. The theory therefore unifies many of the previous approaches. Its application is illustrated by comparison tests, against measurement on prototype motors, against the FE method, and against simple magnetic-circuit models. The relative merits of these methods were discussed and it appears that, although the new analytical methods are more rigorous than previous ones, they are not necessarily faster than the FE method. On the other hand, they are capable of accommodating a wide range of geometric variations without adjustment and without loss of confidence in the result.

\section{REFERENCES}

[1] K. F. Rasmussen, "Analytical prediction of magnetic field from surface mounted permanent magnet motor," in Proc. IEEE IEMDC'99, Seattle, WA, May 9-12, 1999, pp. 34-36.

[2] J. Ofori-Tenkorang and J. H. Lang, "A comparative analysis of torque production in Halbach and conventional surface-mounted permanent magnet synchronous motors," in Conf. Rec. IEEE-IAS Annu. Meeting, 1995, pp. 657-663.

[3] D. Trumper, M. E. Williams, and T. H. Nguyen, "Magnet arrays for synchronous machines," in Conf. Rec. IEEE-IAS Annu. Meeting, 1993, pp. 9-18.

[4] K. Atallah, Z. Q. Zhu, and D. Howe, "Flux waveforms and iron losses in permanent magnet brushless DC machines," in Proc. 12th Int. Workshop Rare-Earth Magnets and Their Applications, Canberra, Australia, July 12-15, 1992, pp. 109-120.

[5] Z. Q. Zhu, D. Howe, E. Bolte, and B. Ackermann, "Instantaneous magnetic field distribution in brushless permanent-magnet dc motors," IEEE Trans. Magn., vol. 29, pp. 124-158, Jan. 1993.

[6] U. Kim and D. K. Lieu, "Magnetic field calculation in permanent magnet motors with rotor eccentricity: With slotting effect considered," IEEE Trans. Magn., vol. 34, pp. 2253-2266, July 1998.

[7] T. J. E. Miller and R. Rabinovici, "Back-EMF waveforms and core losses in brushless DC motors," Proc. IEE-Elecr. Power Applicat., vol. 141, pp. 144-154, May 1994.

[8] T. Sebastian and V. Gangla, "Analysis of induced EMF and torque ripple in a brushless permanent magnet machine," IEEE Trans. Ind. Applicat., vol. 32, pp. 195-200, Jan./Feb. 1996.

[9] N. Boules, "Prediction of no-load flux-density distruibution in permanent-magnet machines," IEEE Trans. Ind. Applicat., vol. IA-21, pp. 633-643, May/June 1985

[10] N. Boules N, "Two-dimensional field analysis of cylindrical machines with permanent magnet excitation," IEEE Trans. Ind. Applicat., vol. IA-20, pp. 1267-1277, Sept./Oct. 1984.

[11] A. Hughes and T. J. E. Miller, "Analysis of fields and inductances in air-cored and iron-cored synchronous machines," Proc. Inst. Elect. Eng., vol. 124, no. 2, pp. 121-126, Feb. 1977.

[12] B. Hague, The Principles of Electromagnetism Applied to Electrical Machines. New York: Dover, 1962.

[13] J. A. Stratton, Electromagnetic Theory. New York: McGraw-Hill, 1941.

[14] T. J. E. Miller, PC-BDC Reference Manual, Version 5. Glasgow, U.K.: SPEED Laboratory, Univ. of Glasgow, June 1999.

[15] T. J. E. Miller, M. I. McGilp, D. A. Staton, and J. J. Bremner, "Calculation of inductance in permanent-magnet dc motors," Proc. IEE-Elect. Power Applicat., vol. 146, no. 2, pp. 129-137, Mar. 1999.

[16] PC-FEA User's Manual, Version 2, SPEED Laboratory, Univ. of Glasgow, Glasgow, U.K., June 1999.

[17] Z. Deng et al., "Fields in permanent magnet linear synchronous machines," IEEE Trans. Magn., vol. MAG-22, pp. 107-112, Mar. 1986.

[18] — - "Forces and parameters of permanent magnet linear synchronous machines," IEEE Trans. Magn., vol. MAG-23, pp. 305-309, Jan. 1987.

[19] - "Analysis of fields and forces in a permanent magnet linear synchronous machine based on the concept of magnetic charge," IEEE Trans. Magn., vol. 25, pp. 2713-2719, May 1989.

[20] _ _ "Fields, forces, and performance equations of air-core liner self-synchronous motor with rectangular current control," IEEE Trans. Magn., vol. 24, pp. 2194-2203, Sept. 1988.

[21] C. S. Koh, H. S. Yoon, K. W. Nam, and H. S. Choi, "Magnetic pole shape optimization of permanent magnet motor for reduction of cogging torque," IEEE Trans. Magn., vol. 33, pp. 1822-1827, Mar. 1997.

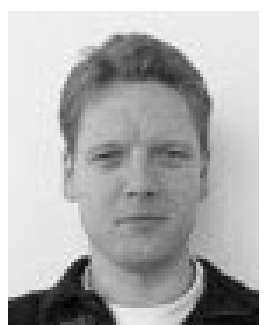

Keld Folsach Rasmussen (S'92-M'95) received the M.S. degree in electrical engineering and the Ph.D. degree from Aalborg University, Aalborg East, Denmark, in 1991 and 1995, respectively.

From 1991 to 1992, he was a Research Assistant with the Institute of Energy Technology, Aalborg University. In 1992, he joined Grundfos A/S, Bjerringbro, Denmark, as an Industrial Ph.D. Student, working with efficiency optimization of electrical machines. In 1995, he joined the Motor Engineering Department at Grundfos, where he is involved with design and control of electrical machines for pump applications.

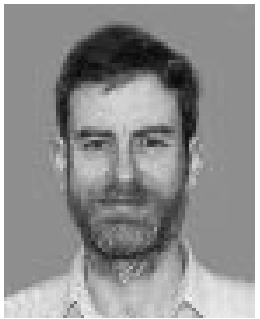

John H. Davies received the Ph.D. degree from the University of Cambridge, Cambridge, U.K., in 1982.

He was a Research Fellow at Cornell University, Ithaca, NY, before joining Glasgow University, Glasgow, U.K., in 1986. He has spent two periods of leave at The Ohio State University, Columbus, and the University of California, Santa Barbara. His research is mainly centered on the physics of transport in III-V heterostructures. This has included the modeling of surfaces and gates, including the effect of stress from patterned surfaces and gates. The work on stress has recently been extended to buried structures, such as self-assembled quantum dots. His other interests include the effect of discrete, random donors on the characteristics of deep submicrometer devices, the theory of resonant tunneling, conduction in lateral superlattices, and the calculation of magnetic fields in permanent-magnet motors.

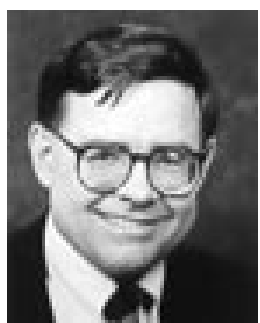

T. J. E. Miller (M'74-SM'82-F'96) is a native of Wigan, U.K. He received the B.Sc. degree from the University of Glasgow, Glasgow, U.K., and the Ph.D. degree from the University of Leeds, Leeds, U.K.

From 1979 to 1986, he was an Electrical Engineer and Program Manager with General Electric Corporate Research and Development, Schenectady, NY. His industrial experience also includes periods with GEC (U.K.), British Gas, International Research and Development, and a student apprenticeship with Tube Investments Ltd. He is currently a Professor of Electrical Power Engineering and founder and Director of the SPEED Consortium, Department of Electronics and Electrical Engineering, University of Glasgow. $\mathrm{He}$ is the author of more than 100 publications in the fields of motors, drives, power systems, and power electronics, including six books.

Prof. Miller is a Fellow of the Royal Society of Edinburgh and the Institution of Electrical Engineers, U.K.

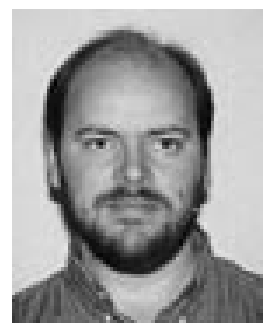

Malcolm lain McGilp was born in Helensburgh, U.K., in 1965. He received the B.Eng. Hons. degree in electronic systems and microcomputer engineering from the University of Glasgow, Glasgow, U.K., in 1987.

Following graduation, he joined the SPEED Laboratory, University of Glasgow, where he was a Research Assistant from 1987 to 1996 and has been a Research Associate since 1996. He is responsible for the software architecture of the SPEED Laboratory motor design software and has developed the interface and user facilities which allow ease of learning and integration with other PC-based software. 


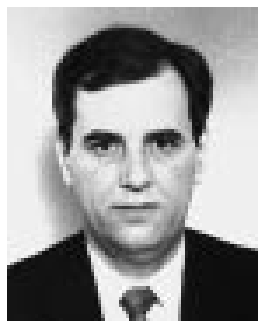

Mircea Olaru studied in the Faculty of Mechanics-Mathematics, Bucharest University, Bucharest, Romania.

$\mathrm{He}$ is currently an Analyst and Programmer with ICNDMF-CEFIN, Bucharest, Romania, where he works with research programs in the mechanical field. He has authored 35 published articles and has presented 54 essays at national and international scientific meetings. 